\title{
Prokla-Redaktion \\ Editorial: Das politische Problem der Generationen
}

Das politische Problem der Generationen beginnt dort, wo eine Generation aufhört. Sechs von neun Redaktionsmitgliedern der PROKLA gehören den Jahrgängen an, die sich jetzt auch die Sozialforscher angewöhnt haben, »Protestgeneration « zu nennen, während die drei verbleibenden zu jener unglücklichen Kohorte zählen, die schlicht nur als »verloren « gilt. Die »lost generation« kommt alle Jahre mal vor und hat im 20 . Jahrhundert eine reiche Tradition. Nicht nur die, die klagen, für ' 68 zu jung und für den Punk zu alt gewesen zu sein, suhlen sich gerne in der Melancholie, das Wesentliche verpaßt zu haben. So sangen schon die Alten, als sie noch kurze Hosen trugen, und später dann haben sich junge Männer dieses Gefühl beim Rock'n'Roll aus dem Körper getanzt. Verloren waren diese Generationen durch den Krieg, die späteren durch das Empfinden, nicht überall dabeigewesen zu sein.

Im Unterschied zu den »verlorenen « haben die »politischen « Generationen immer schon einen Fixpunkt außer sich selbst gehabt. Träger einer Idee und einer Bewegung zu sein, erweitert das kollektive Selbst um politische und soziale Dimensionen, die durch keine Jahrgangsgrenzen mehr beschränkt scheinen. Die politischen Brüche in der deutschen Geschichte, die Verbrechen, Katastrophen und Errungenschaften, die sie hervorgebracht hat, lassen darauf ein anderes Licht fallen. Die politische Geschichte Deutschlands ist eine Geschichte der Brüche zwischen den Generationen. Vom »jungen Deutschland « bis zur »deutschen Jugend «, von der »Frontkämpfergeneration « bis zur Hitlerjugend, von der skeptischen »Ohne-mich-Generation« bis zu den »68ern«, von den »alten Genossen « der Komintern-Zeit bis zur FDJ-Generation der DDR-Aufbaujahre haben sich Jahrgangsgruppen als Widerpart zu anderen im Auftrag höherer Ziele verstanden. Nach 1968 sind die Wellen, nach denen politische Generationen bezeichnet wurden, immer spärlicher an das Ufer des öffentlichen Bewußtseins geschlagen - vielleicht weil es sie so seither nicht mehr gab. Ein Label ist schnell kreiert und muß bei weitem nicht zutreffend sein - auch Schelskys »Skeptische Generation « der 50er Jahre stammt nicht von ihm, sondern von seinem Verleger. Von denen, die zwischen 1940 und 1950 geboren sind, waren nur wenige 1968 wirklich dabei. Doch hat sich der Zeitgeist dieser Epoche in welchen Färbungen auch immer im Bewußtsein dieser Bevölkerungsgruppe abgelagert, hat sich ein »Generationsstil « (Karl Mannheim) entwickelt, der untereinander ebensosehr Erkennbarkeit stiftet, wie zwischen den Generationen für den Anlaß von Absetzbewegungen sorgt. Mag sein, daß es die politischen Generationen gar nicht (mehr) gibt, mag auch sein, daß das Gerede von den Verwerfungen der eigenen Generationszugehörigkeit ein typisches Syndrom der Mentalität der Mittelklassen ist. Und doch ist die Frage, in welchen Altersgruppen die Problematik einer Zeit am heftigsten empfunden wird, nicht nur ein Thema für verregnete Nachmittage. Wahrnehmung und Bewußtsein 
steuert sich nicht jenseits des eigenen Alters aus und kollektives politisches Handeln begründet sich am ehesten in Lebenswelten, wie sie nicht nur von einer Klasse, sondern auch in einer Generationenlage gemeinsam erlebt werden. Als Karl Mannheim 1928 das »Problem der Generationen« beschrieb, wird er die »alten Kämpfer«, die »neue Frau « und die »deutsche Jugend « vor Augen gehabt haben - soziale Typen generativer Einheiten, zwischen denen die kulturellen und politischen Konflikte der Zeit ausgetragen wurden. Dabei fällt auf, daß politische Generationen eigentlich immer jung waren, jedenfalls sind es immer nur die Jüngeren gewesen, die so bezeichnet wurden. Die formative Phase des politischen Bewußtseins pflegt einzusetzen, wenn die Gesellschaft zum ersten Mal bewußt und praktisch angeeignet wird und dabei die Ordnung der Welt noch bar jeder Normalität ist. Das wußte schon der Hegelsche Jüngling, so wie ihn Manfred Riedel beschreibt:

«Der Jüngling ... Iöst die in der Welt und ihren ethisch-politischen Dauerordnungen von Familie, bürgerlicher Gesellschaft und Staat verwirklichte sittliche Idee auf die Weise auf, daß er sich selber die zur Natur der Idee gehörende Bestimmung des Substantiellen - das Wahre und Gute -, der Welt dagegen die Bestimmung des Zufälligen, Akzidentiellen zuschreibt.«

Generationen erleben eine historische Aktualität, die für andere Vergangenheit ist. Gleichzeitiges erleben sie ungleichzeitig, als primäre Erfahrung, als Kontinuitätsbruch, Enttäuschung oder Bekräftigung. Generationen durchlaufen eine altersbedingte Prägephase ihres politischen Bewußtseins (die »Jahre der Empfänglichkeit«, wie Dilthey das genannt hat), ihre Weltbilder und Mentalitäten formen sich in der Ersterfahrung der je historischen Ereignisse einer bestimmten Zeit, was die gemeinsame Jugend für das eigene Generationsgefühl besonders prädestiniert. Politisch werden Generationen immer dann, wenn diejenigen, die verwandten Geburtsjahrgängen angehören, die typischen Ereignisse ihrer Zeit in gleicher Weise verarbeiten und ihr politisches Handeln mit gemeinsamen Grundintentionen und Zielen ausstatten. Das heißt nicht, daß Generationen handelnde Gruppen sind, wohl aber, daß handelnde Gruppen durch das Merkmal der Generationszugehörigkeit geprägt sind. Weil Alte weniger aktiv sind und Vierzigjährige zu tun haben, sieht es so aus, als ob dies nur die Jüngeren betrifft. Das auch politische Pathos der Jugend verstellt den Blick darauf, daß es vor allem die mittlere Generation ist, die eine Gesellschaft trägt, die Generation der Kader und Familienväter, der Stammbelegschaften, der Bürokratie und der Führungskräfte. Politisch ist diese Generation immer schon durch die Funktion, die ihr im sozialen Gefüge zukommt, und politisch ist sie auch dadurch, daß sie die Themen, Leitbilder und gesellschaftlichen Regeln, die Auf- und Abstiegsbedingungen vorgibt, die die Spannung zu den nachwachsenden Altersgruppen erzeugen.

Wie der sozialstaatlich gebändigte Kapitalismus durch Arbeitsmarkt, Ausbildung und Verrentung den Altersaufbau einer Gesellschaft erst zu einer sozialen Struktur verfestigte, so ist auch die »Generation« ein Begriff, der höchstens zweihundert Jahre hinter sich hat. Auf diesem Weg ist er von den literarischen Salons, wo er zur Selbstbeschreibung einer ästhetischen Elite diente, in die Arenen der Politik gewandert, um schließlich von der Reklame entdeckt zu werden - was für seine Realitätstüchtigkeit spricht: Kein Kapitalist gibt sein Geld für unnützes Zeug aus. Gleichwohl schwebt 
regelmäßig ideologischer Nebel durch den Raum, wenn von Generationen die Rede ist - harmlos, sentimental, harmonisierend.

Generationen sind keine homogenen sozialen Einheiten. Mannheim meinte, den Generationenbegriff nur deshalb zum Klassenbegriff analog setzen zu können, weil ähnlich der »Klasse an sich« die Generationslagerung die strukturell-historischen Bedingungen vorgibt, unter denen Praxis möglich ist, Welt und Gesellschaft erfahren und verändert werden kann. Die Generationszugehörigkeit lagert sich in die jeweilige Klassenlage ein, modifiziert das Erleben und die Handlungsbereitschaft. Die wirklichen Träger sozialer Bewegungen sind nicht nur sozial, sondern oft auch generativ miteinander verbunden gewesen. Darin liegt die Stärke der inneren Kohärenz einer Bewegung begründet, aber auch die Schwäche der Schließung zu anderen Altersgruppen. Das politische Problem der Generation beginnt hier, in der Vermittlung in Form einer gemeinsamen Sprache, in der Schwierigkeit, die Kultur eines Protestes und seiner Ziele in andere Altersgruppen transformieren zu können. Mannheim sah die praktische Bedeutung des Generationenproblems darin, in Zeiten »beschleunigter Umwälzungen ein genaueres Verständnis der unmittelbaren Gegenwart « zu erlangen. Wenn Krieg oder Klassenkampf, Depression oder Revolution die historische Kontinuität durchbricht, setzt der geschichtliche Wandel vor allem die Kräfte, das Engagement und die Ambitionen der nachrückenden Generationen frei, die den politischen Prozeß mit einer besonderen Intensität erleben. Im historischen Augenblick der Veränderung sind durch die Klassen hindurch Generationsgruppen die Träger einer politischen Bewegung, sind politische Mentalitäten und auch materielle Interessen nach Altersgruppen differenziert, und wer deren Motive und Erfahrungen kennt, wird Ursachen und Chancen politischer Umwälzungen besser einschätzen können. Wenn einer bald sein ganzes Leben damit verbracht hat, sich anpassungsbereit zu zeigen, wird er in die Zukunft kein politisches Risiko mehr investieren wollen. Nichts zu verlieren haben nur die, die sich in der Herrschaft noch nicht einrichten konnten. Die, die sich arrangiert hatten, bemühen sich nach einer Umwälzung meist, ihre vormalige Konformität als lebenslangen inneren Widerstand auszugeben. Wir kennen das aus der Geschichte, zuletzt aus der DDR. Die »Hoch «-Rufe vor den Tribünen des Politbüros mögen in manchem noch nachgehallt haben, der nach dem Sturz der Nomenklatura seinen Abscheu darïber bekundete, die ganze Zeit über betrogen worden zu sein. Die Kinder wußten das besser und zogen ihre Schlüsse daraus: sie votierten für »exit« oder »voice«, während etabliertere Jahrgänge bis zum 9. November erst einmal abwarteten, wer am Ende die besseren Karten hat.

Das politische Problem der Generationen liegt daher auch im materiellen und moralischen Erbe begründet, das eine den anderen überläßt. Die Protestgeneration der sechziger und siebziger Jahre hat versucht, die satte Zufriedenheit des Postfaschismus mit sich selbst aufzuscheuchen. In der DDR mußte das herrschende Bündnis aus alten kommunistischen Kadern und den willigen Technokraten der mittleren Generation erfahren, daß nach dem letzten Tauwetter zu Beginn der siebziger Jahre der Faden zu den nachwachsenden Altersgruppen langsam aber sicher gerissen war. Auf der Baustelle des Sozialismus setzte seither eine innere Kündigung ein, die nur auf die Gele- 
genheit wartete, dem ungeliebten Regime Hammer, Zirkel und Ährenkranz endlich hinschmeißen zu können. Nicht nur für die Veteranen der Arbeiterbewegung ist dies eine schmerzhafte Erfahrung gewesen, in die sich auch Scham mischte, jedenfalls bei denen, die dem Zynismus der Macht nicht vollends erlegen waren. Oftmals waren es gerade die Dissidenten des Sozialismus, die in eine tiefe Depression gefallen sind, als es nach dem November in den Bildern des Fernsehprogramms nur noch Deutschland total gab. Der Ideale beraubt und für all die Konflikte, die man ihnen zuliebe auf sich nahm, nicht im geringsten entschädigt: tabula rasa des eigenen Selbst - so jedenfalls will die Geschichte es anscheinend lehren, die mit ihrer ganzen Gewalt über die eigenen Normen hinweggerollt ist und der eigenen Biographie den Stempel der Vergeblichkeit aufgedriickt hat.

Zum Generationenthema hat die Linke in diesem Land seit '68 ein gestörtes Verhältnis gehabt, nicht ohne Grund, aber auch nicht ohne Verkennung. Zu den begründeten Aversionen gehört, daß es sich noch jeder politische Aufbruch gefallen lassen mußte, als Generationenkonflikt « verniedlicht zu werden: »alles schon mal da gewesen «. Der Verkennung muß zugerechnet werden, daß der Generationenkonflikt von denen, die ihn betrieben haben, selbst immer nur vulgärpsychologisch verstanden wurde. $\mathrm{Da}$ sich politische Mentalitäten um Generationsgruppen bündeln und mit dem Alter verbunden sind, sollte nicht eingestanden werden, um die Universalität der Idee nicht zu blamieren. Die Fakten dagegen sind kalt und nüchtern. Die Wahlforschung etwa hat die GRÜNEN schon längst als Kohortenpartei der 30 bis 45jährigen ausgemacht, weshalb mit ihrem politischen Fortbestand zu rechnen sei, auch wenn der Anteil der Jungwähler weiterhin sinkt. Weil mit zunehmendem Alter die politische Wahlpräferenz immer weniger noch verändert wird, die politische Grundposition sich also biographisch verfestigt, wandert die Wählerschaft mit den Jahrgangsklassen in die oberen demographischen Abteilungen ab, was bei der derzeit durchschnittlichen Lebenserwartung den GRÜNEN noch auf 30 bis 40 Jahre eine Stammwählerschaft bescheren könnte, die das Erreichen der 5 Prozent-Klausel nicht unwahrscheinlich macht.

Heute befindet sich die Linke in einer Generationenspannung, aus der sie selbst einmal hervorgegangen ist, nur daß sich mittlerweile die Rollen vertauscht haben. Die Töchter der Emanzen und die Söhne der 68er treten zum Bedauern ihrer Eltern nicht in die linke Geschichte ein, sondern - so hört man es jedenfalls - profitieren nur kaltschnäuzig von jenen materiellen Vorteilen und kulturellen Freiheiten, die andere für sie einmal erkämpft haben. Die Hymne einer Generation schien gewechselt zu haben: von »Keine Macht für niemand « zu »Flieger, grüß mir die Sonne«-der linke Lehrer schier verzweifelt, was für eine spießige oder auch nur banale Brut er da herangezüchtet hat. Die Selbstgerechtigkeit der alternden Rebellen ist mindestens so ätzend wie die schick gewordene Bereitschaft, nun unbedingt alles toll finden zu müssen, was der junge Mensch so treibt. Dazwischen liegt der wirkliche Prozeß, sich zwischen Überheblichkeit und fürsorglicher Belagerung sowohl absetzen als auch verstehen zu können. In der Bundesrepublik liegen heute die Erfahrungsbestände zwischen der mittleren und der jungen Generation vielleicht dichter zusammen, als dies je zuvor der Fall 
gewesen ist. Man kann miteinander reden, und daß eine 40jährige Mutter mit ihrem dreizehnjährigen Sohn gemeinsam ein Konzert von Prince besucht, dürfte vor zwanzig Jahren bei vergleichbaren Anlässen keineswegs gewöhnlich, sondern eher eigenartig gewesen sein. Die mittlere Generation der Bundesrepublik ist eine, die selbst schon eine verlängerte Jugend gehabt hat und die das Erwachsensein nicht bruchlos erfuhr, nicht kulturell, aber auch nicht ökonomisch. Dafür hat der Arbeitsmarkt gesorgt, der die Statuspassagen unsicherer machte. Mancher kam dadurch auf den blöden Gedanken, daß es »sinnlos sei, erwachsen zu werden. «Vergleichbares war aus der DDR 40 Jahre lang nicht zu berichten, wo der vormundschaftliche Staat den Bürger als Mündel hielt und ihm bei Gehorsam die realsozialistische Variante der staatlichen Vollversorgung bot. Der Umgang mit Unsicherheit ist dadurch auch für die verschiedenen Generationen der DDR zu einem Thema geworden, und wohl und wehe denen, die sich an ihre Erwartungen noch nicht allzu oder schon zu sehr gewöhnt haben. In der DDR hat es ein 1968 nicht gegeben, die Widerstände gegen die alte Ordnung haben sich andere Daten und Themen gesucht. Die Jugend der achtziger Jahre hat den anpassungsbereiten Aufbaugenerationen des realen Sozialismus politisch Beine gemacht, um schließlich auch in der eigenen Altersgruppe mit einem Thema konfrontiert zu werden, das scheinbar einer anderen Generationenlage angehört: der Nation. Generationen haben ihre politischen Themen, um die herum sich das politische Bewußtsein zentriert. Faschismus und Technik sind hier Stichworte gewesen, ob Deutschland wieder eines werden wird, ist gegenwärtig noch nicht ausgemacht. Merkwürdig verzerrt erscheinen politische Themen immer dann, wenn sie ganz einfach nicht zum Alter passen wollen. Daß jene, die in einem Deutschland aufgewachsen sind, hieran besonders hängen, mag noch verständlich sein. Daß aber auch Menschen für Schwarz-Rot-Gold zu begeistern sind, die allein von ihrem Alter her damit doch keine konkrete Vorstellung verbinden können, hat nicht mit der Erfahrung, sondern mit einer Projektion zu tun - und derlei ist politisch immer viel gefährlicher geladen, als Erfahrungen dies je sein können. Die Umwälzungen in der DDR können aber auch zu anderen beunruhigenden Wahrnehmungen veranlassen, die mit den politischen Haltungen verschiedener Generationen zu tun haben. Während die »verdorbenen Greise « der stalinistischen Gerontokratie den Sozialismus auf eine billige Legitimationsideologie herunterbrachten, hat sich eben dadurch die Idee des Sozialismus für andere Generationen restlos verbraucht. In ihren geschichtlichen Erfahrungsbeständen, die hier vom Faschismus, dort von der Schurigelei, der Langeweile und der Bevormundung geprägt waren, vollständig getrennt, herrscht Verachtung zwischen den Generationen, kalte Wut und Sprachlosigkeit. Wenn der Sozialismus selbst nicht zu einer historischen Reminiszenz einiger Generationen werden will, wird er eine neue politische Generation für sich erobern müssen. 